BMJ Open

Sport \&

Exercise

Medicine

\title{
Capturing effort and recovery: reactive and recuperative cortisol responses to competition in well-trained rowers
}

\author{
Victor L Kallen, ${ }^{1}$ Janine H Stubbe, ${ }^{2,3}$ Henk Jan Zwolle, ${ }^{4}$ Pierre Valk ${ }^{1}$
}

To cite: Kallen VL, Stubbe JH, Zwolle HJ, et al. Capturing effort and recovery: reactive and recuperative cortisol responses to competition in well-trained rowers. BMJ Open Sport Exerc Med 2017;3:e000235 doi:10.1136/bmjsem-2017000235

- Additional material is published online only. To view please visit the journal online (http://dx.doi.org/ 10.1136/bmjsem-2017000235).

Accepted 26 May 2017

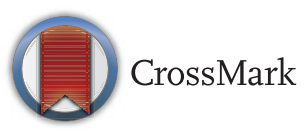

${ }^{1}$ The Netherlands Organization for Applied Scientific Research (TNO), Zeist, The Netherlands ${ }^{2}$ Amsterdam University of Applied Sciences, School of Sports \& Nutrition, Amsterdam, The Netherlands ${ }^{3}$ Codarts University of the Arts, Rotterdam, The

Netherlands

${ }^{4} \mathrm{HJZ}$ Sport, The

Performance Experts,

Amsterdam, The Netherlands

\section{Correspondence to}

Victor L Kallen, The

Netherlands Organisation for Applied Scientific Research (TNO), Utrechtseweg 48, P. 0. box 360, 3700 AJ Zeist, The Netherlands, ; victor. kallen@tno.nl

\section{ABSTRACT}

Background/aim It is well known that physical strain is associated with increased cortisol production. And although mental stress elevates cortisol concentrations as well, little is known of the independent and/or combined effects of both on the secretion of cortisol. Aim of the study was to investigate the day-to-day cortisol dynamics associated with training, performance and recuperation and the immediate responses to mental stress and physical endurance under competitive conditions.

Methods Sixteen freshmen competitive male rowers were prospectively followed from Thursday to Tuesday with an intermediate competition on Saturday and Sunday. On all days, three saliva samples were collected within $30 \mathrm{~min}$ after awakening to assess the cortisol awakening rise (CAR). Additionally, five saliva samples were collected previously to and immediately after all races during the regatta weekend.

Results CAR values peaked during competition days and recovered during the 2 days after. Cortisol concentrations significantly increased during and after all races. Furthermore, although response patterns did not differ, the morning races showed significantly higher cortisol levels compared with the levels measured during the afternoon races. This likely reflects the normal diurnal rhythm of corticosteroids.

Conclusions These results indicate that cortisol levels of athletes might be sensitive for both immediate responses to competition and, in case of CAR, (midterm) recovery phasing. Consequently, monitoring cortisol responses during training and competition may provide valuable information regarding how athletes cope with competition-induced stress and their recovery status during the days following. This insight might help to plan future training loads and recovery.

\section{INTRODUCTION}

Athletes are constantly exposed to a wide range of stressors. ${ }^{1}$ Daily training load is the key source of physiological stress in athletes. ${ }^{2}$ Intensity and duration of endurance exercise is positively related to cortisol elevation in the human body. ${ }^{3-12}$ Furthermore, in official competitions, athletes are submitted to a wide range of psychological stressors, such as pressure to achieve optimal outcomes, the unpredictable

\section{What are the new findings}

Monitoring cortisol responses during training periods and competition using standardised methods (eg, cortisol awakening rise) seems to provide valuable information regarding how athletes or oarsmen cope with competitioninduced stress. This insight might help to plan future training loads and recovery.

- Additionally, our findings seem to emphasise that coaches should avoid excessive training loads for at least 2 days after competition, because the physical and mental recuperation process (as reflected in hypothalamic-pituitaryadrenal axis functioning) might still in progress by then.

- This finding might be an argument for the use of psychological strategies to improve the coping skills of rowers to deal with the additional stress inherent to rowing events, thus potentially accelerating the overall recuperation process.

environment related to official matches and the importance of the match. ${ }^{2}$ These stressors might lead to negative affective states, ${ }^{13}$ which can result in increased levels of cortisol.

The degree and duration of the elevation of cortisol are considered indicative of stress. Mild increases in cortisol prepare individuals for action and lower cortisol levels or reactivity may indicate more resilience to stressful situations. On the contrary, extreme elevations in cortisol lead to poor performance, because it interferes with some cognitive processes and may suppress testosterone production. ${ }^{14}$ The cortisol awakening rise (CAR) is an established marker of more chronic and pervasive (either mental or physical) stress, for example, during the previous days to weeks. ${ }^{15} 16$ To our knowledge, no studies in rowing have investigated whether the endurance and recuperation status might be mirrored in measurable (significant) day-today alternations in CAR. Therefore, the 
first aim of our study was to investigate the day-to-day cortisol dynamics associated with training, performance and recuperation in oarsmen.

Furthermore, significant higher cortisol levels have been identified in competition versus simulated events in many different types of sports (eg, fighting sports like jiu-jitsu and judo, tennis and weightlifting). ${ }^{10} 17-19$ Studies in rowing investigated the effect of cortisol in response to various types of training and periodisation. ${ }^{20-22}$ To our knowledge, only one study has examined the effects of competition compared with training on the cortisol concentrations of rowers, ${ }^{23}$ and none using a repeated measurement design incorporating sequential samples immediately previous to and following a competitive event. Pearson et $a l^{23}$ collected urinary cortisol levels in two teams of Oxford college eights oarsmen. These were compared on training days, racing days and non-rowing days. Results showed that (urinary) cortisol levels were elevated on racing and training days compared with non-racing days. One disadvantage of this study is that it by design used a wide time slot to measure cortisol levels: total urine samples were collected over a 3.5-hour period, whereas the race itself could take no more than $5-6 \mathrm{~min} .{ }^{23}$ It is unclear whether cortisol levels were measured before or after the races. Therefore, the second aim of our study was to investigate the immediate effect of competition on cortisol levels in oarsmen.

\section{MATERIALS AND METHODS}

\section{Subjects}

Two rowing crews (freshmen's eights) were included in this study, overall including 16 competitive male rowers: one 'lightweight' eight $(\mathrm{n}=8$, average weight $70 \mathrm{~kg}$, no individual rower over $72.5 \mathrm{~kg}$ ) and one 'open class' eight $(\mathrm{n}=8$, no weight restrictions). The coxswains of both crews assisted in applying the study protocol, though they did not collect data themselves.

Before participation, all rowers were medically cleared according to the Lausanne recommendations ${ }^{24}$ and all rowers signed an informed consent. The Institutional Review Board of TNO granted ethical approval for the study. All participants finished the study and received a monetary reward afterwards.

Rowers in the Dutch freshmen's competition generally start with approximately four training sessions per week as from September. From January, training intensity is increased to on average of six training sessions every week. The regatta season starts in the beginning of April and ends in the first weekend of July.

Each regatta consists of four races: both on Saturday and Sunday a preliminary and (if a crew indeed qualifies) a final. In a typical rowing race, up to six crews race side-by-side, each in their own lane, over $2000 \mathrm{~m}$. The type of crew participating in the present study generally needs (depending on the weather) 6-7 min to complete the race. During the present study, weather conditions were generally good (dry, comfortable temperatures and not too much wind).

There is a regatta every other week, which means that generally a regatta is followed by a recovery week. In this week, the focus is on physiological recovery implying a more extensive training load. The last days before the regatta training sessions are relatively short though intensive, for example, to train tactical situations like starts and sprints.

\section{Procedures}

This study was conducted mid-season: in the first week of June 2011. Rowers were prospectively followed during oneweek from Thursday to Tuesday. In this week, the regatta was held on Saturday and Sunday, conveniently on their own training waters. On Saturday morning, both crews rowed a preliminary race shortly after each other and finals late in the afternoon; on Sunday, the preliminaries were clearly on separate times (heavyweight crew at 08:00 hours; lightweights shortly after 12:00 hours). The lightweight eight did not qualify for the finals on Sunday. They nevertheless collected saliva samples on the times when they would have raced the finals, thus providing unanticipated baseline cortisol saliva values (see figure $1 \mathrm{~B}$, online supplementary figure $1 \mathrm{~A}, \mathrm{~B}$ ).

To be able to collect (cortisol) data, the Royal Dutch Rowing Association (KNRB) provided a testing room at the national training centre, being close by the boathouse of the participating rowers and at the shores of the regatta track.

Saliva samples were collected on six consecutive mornings (Thursday to Tuesday) immediately after awakening (07:00, 07:15 and 07:30 hours) using Salivettes (Sarstedt, Germany). The collected samples were immediately brought to the testing room at the training site where they were stored at $-20^{\circ} \mathrm{C}$. These data were used to calculate for each rower a daily CAR, being the cumulative of the three values. Average CAR per crew (lightweight vs heavyweight) was calculated for each day: Thursday and Friday previously to the upcoming competition; Saturday and Sunday being the competition days and Monday and Tuesday, postcompetition recovery days.

Additionally, on competition days (Saturday and Sunday: seven races overall), additional saliva samples were collected $30 \mathrm{~min}$ before boarding; immediately before boarding (after mental preparation, though before any serious physical exercise); after warming up in the starting zone; immediately after the finish of the race and 20 min later after standardised cooling down in the boat. After data collection was completed, all samples were transported to the laboratories of the Academic Hospital Utrecht (U-Diagnostics, UMC Utrecht, The Netherlands) to be analysed by means of radioimmunoassays (Coat-A-Count; Diagnostic Products, Los Angeles, California, USA): lower detection 
A.

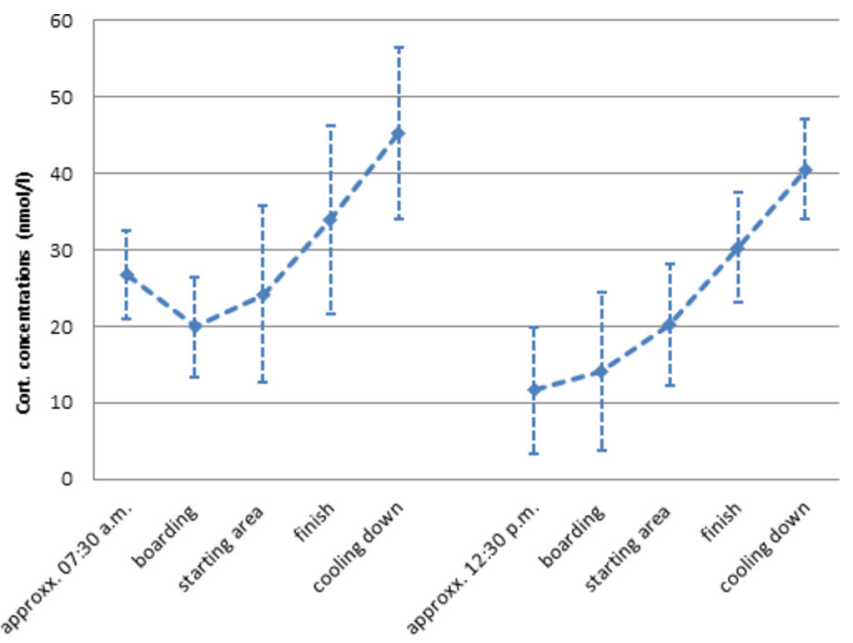

B.

Cortisol concentrations light weights, Sunday: preliminary only

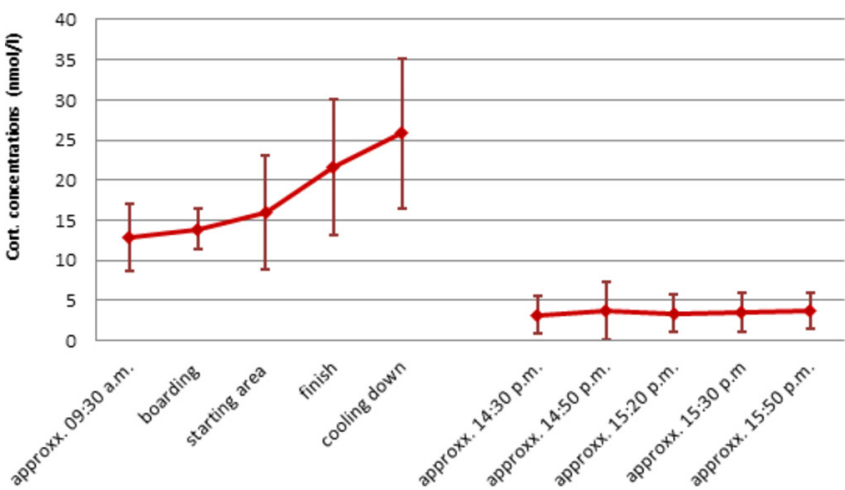

Figure 1 The heavyweights on Sunday (competition day 2): average cortisol concentrations with SD (vertical bars) indicated previously to and immediately after the races. The heavyweight eights $(n=8)$ rowed the preliminaries (in the morning) and qualified for the finals of their division (in the early afternoon, contrary to the lightweights $(n=8)$ ). The lightweights on Sunday (competition day 2): average cortisol concentrations with SDs indicated. The lightweight eight $(n=8)$ rowed their preliminary in the Dutch lightweight elite division (in the morning) and failed to qualify for the afternoon finals. Consequently, the average afternoon cortisol levels of this crew can be considered a reliable baseline and are significantly lower than the other assessed (prerace and postrace) values (for both crews).

limit of $1 \mathrm{nmol} / \mathrm{L}$; intra-assay variation of $4.4 \%$ and an interassay variation of $5.2 \%$.

Of the 608 collected samples, only eight were either missing or contained too little saliva to be able to reliably derive cortisol concentrations from them.

The heavyweight crew classified on both days for the finals (consequently rowing four races in 2 days), whereas the lightweight crew won Saturdays finals (against the other freshmen's eights), though lost Sunday morning's preliminary to a Dutch national crew. Consequently, the lightweight crew only rowed three races this weekend; however, this provided an excellent post hoc (Sunday afternoon) baseline for reference, as they all remained at the regatta course and collected saliva samples at the appropriate times (as if they would have competed in the finals, see figure $1 \mathrm{~B})$.

\section{Statistical analysis}

The CAR was calculated for each individual rower and averaged over both crews for each assessment day. Analyses of variance (repeated measures) were used to investigate changing CAR over the sequential days, with post hoc t-tests to identify potential significant differences on a day-to-day basis.

Saliva cortisol concentrations previously to, during and immediately following each race were analysed using repeated measurement analyses of variance, with post hoc F-test to investigate potential difference between individual assessments, either within or between crews. Data processing and analyses were done using Microsoft Excel and SPSS. Alpha's smaller than 0.05 were considered 'significant'.

\section{RESULTS}

CARs per day are presented in figure 2, Supplementary file 2. The CAR data of one heavyweight rower were excluded in the analyses because he had significantly lower morning cortisol values than all other participants (<3 SD from overall average). Overall CAR values changed significantly over the complete 6-day period $(\mathrm{F}(5,9)=4.85, \mathrm{p}=0.02)$. However post hoc contrasts found no significant differences from day-today: While the heavyweight crew showed an overall decreasing trend in morning cortisol concentrations over the days, the lightweight crew seemed to peak with a higher CAR on Sunday morning as compared with the heavyweights as well, being reflected in a trend towards a significant difference in CAR ( $\mathrm{t}(11.3)$ $=-1.93, \mathrm{p}=0.08)$. Overall CAR development seemed to be best captured in quadratic terms $(\mathrm{F}(1,14)=13.12$, $\mathrm{p}=0.003)$ suggesting cortisol morning values to peak during, or shortly after, the regatta weekend.

Figure 3A, B show the preliminary and final results for the races on Saturday morning and afternoon, respectively. Over both the preliminaries ( $\mathrm{F}$ $(4,15)=11.11, \mathrm{p}<0.0005)$ and finals $(\mathrm{F}(4,15)=55.65$, $\mathrm{p}<0.0005)$ cortisol concentrations increased significantly. Furthermore, comparing the cortisol levels of the Saturday morning and afternoon races showed a significant higher overall cortisol level in the morning race for both crews $(p=0.04$, twotailed test), supplementary figure 3 . 


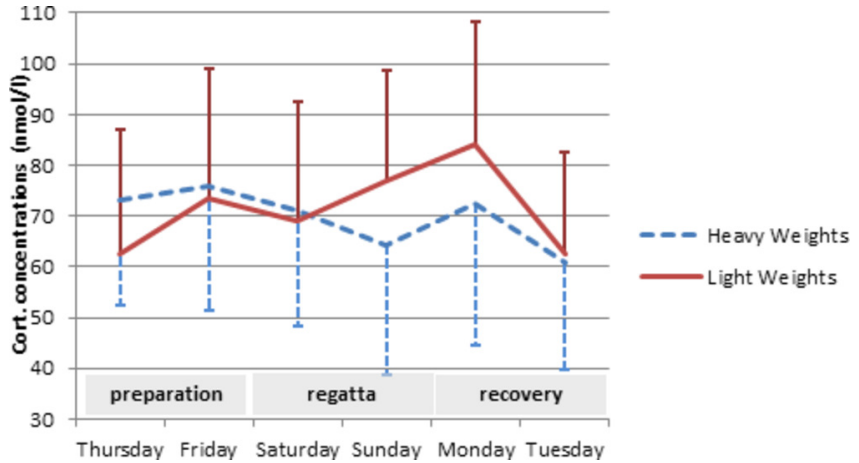

Figure 2 Average cortisol awakening rise for each assessment day, per crew (heavyweights: $n=7$; lightweights: $n=8$ ) with SD (indicated by the vertical bars; heavyweights down, lightweights up). Thursday and Friday in anticipation of the regatta weekend; Saturday and Sunday being the competition days and Monday and Tuesday the recovery stage.

A.

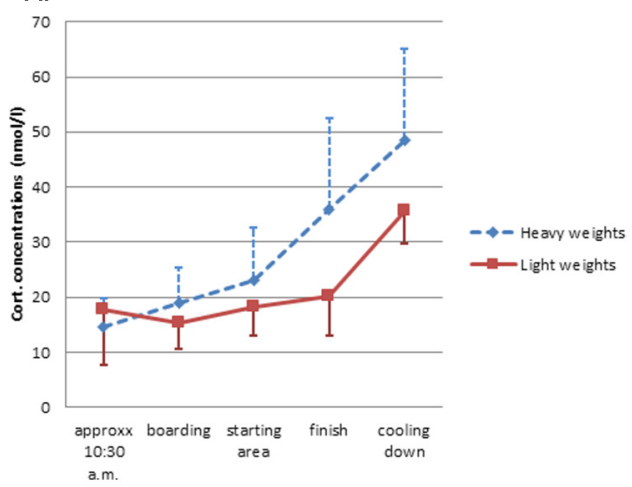

B.

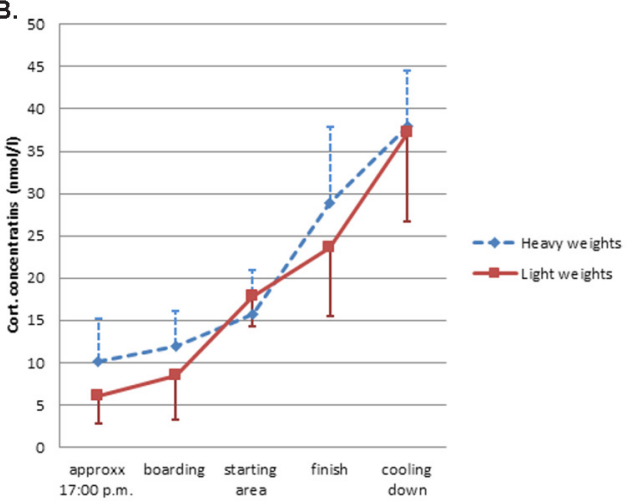

Figure 3 (A) Average cortisol concentrations with standard deviations (indicated by the vertical bars; heavy weights $(\mathrm{N}=8)$ upward direction; light weights down $(\mathrm{N}=8)$ ) previously to and immediately after the preliminary races on Saturday (competition day 1). Cortisol concentrations increased significantly $(F(4,15)=11.11, p<0.0005)$. (B) Average cortisol concentrations with standard deviations (indicated by the vertical bars; heavy weights $(\mathrm{N}=8)$ upward direction, light weights down $(\mathrm{N}=8)$ previously to and immediately after the finals on Saturday (competition day 1). Cortisol concentrations increased significantly $(F(4,15)=$ 55.65, $\mathrm{p}<0.0005)$.

Cortisol data of Sunday (competition day 2) is presented in figure 1A (heavyweights) and figure 1B (lightweights): two races of the heavyweights, only preliminaries for the lightweights, with normal (nonracing) afternoon values. Again, a significant increase was found in cortisol concentrations around the preliminaries $(\mathrm{F}(4,15)=24.84, \mathrm{p}<0.0005)$. In addition, due to the fact that the crew did not qualify for the finals on Sunday, we were able to quantify the race effect in corticosteroid production.

The prerace cortisol samples of the lightweight crew did not differ significantly between the Saturday and Sunday afternoon nor did it between the first (lightweight prerace) samples of Sunday morning and nonrace samples of Sunday afternoon (all p's $>0.1$ ). However, the difference between the last postrace concentrations after Saturday finals and the non-race concentrations on Sunday afternoon (the latter five data points showed no significant change over time (as to be expected, see figure 1B)) were highly significant $(\mathrm{p}=<0.0005)$. Compared with the heavyweights, on Sunday afternoon, the differences between both the first (prerace) cortisol values $(\mathrm{p}<.005)$ and the last postrace values $(\mathrm{p}<0.05)$ were all significant, with the heavyweights having the higher cortisol concentrations (see figure 1A, B).

Finally, the postrace Sunday afternoon differences between both crews seem to make utter sense from the obvious discrepancy in the physical workload provided by both crews (racing vs totally idle).

\section{DISCUSSION}

In this study, we assessed the salivary cortisol responses to competition and postcompetition recuperation in well-trained rowers. First, we found that 2 days after the regatta competition, early morning cortisol levels were recovered to precompetition levels. This finding seems to be in accordance with Pearson and colleagues, who also reported higher overall cortisol levels during race days compared with non-rowing days. ${ }^{23}$ Potential 
(statistical) differences between both studies might likely be explained by the fact that the study by Pearson and colleagues covered wider time slots as they collected urine samples instead of series of eventrelated consecutive saliva samples. ${ }^{23}$ Allthough urine samples are an established assessment of hormonal parameters, it typically measures the production of cortisol over longer periods of time (ie, the overall production within the interval between two consecutive urinations), and is consequently much less able to capture potentially relavant dynamic/event-related corticosteroid responses. However, capturing these relatively rapid responses (like the cortisol awakening response) is very well possible using well timed saliva samples. As a result, our findings provide additional evidence that the physical recuperation process might very well be still in progress 2 days after the competitive event, suggesting that training load may have to be reduced for at least 2 days after competition. If early morning cortisol values are considered a trait marker for available (energetic) resources (being over time the inverse of experienced stress, either mental and/or physical), ${ }^{15} 16$ this may explain the (distinct) CAR patterns we found in both crews: whereas the lightweights won Saturdays final (followed by a 'cortisol boost' on Sunday and Monday morning), the heavyweights against expectations lost the Sunday final. Given the steadily decreasing trend in morning cortisol values in the heavyweight crew, it may be speculated that this crew was yet in a process of recuperation and consequently not fit enough on Sunday afternoon. Though more research seems necessary to shed more light on long term ( $>1$ week) phasing in corticosteroid recuperation dynamics.

Our second finding showed that significant higher concentrations of cortisol were found after the races compared with the levels prior to the races. We can only compare these results with findings in other sports, because to our knowledge no other study has investigated this research question in rowing. A study focusing on a kumdo (kendo) team competition showed significant higher cortisol levels in postcompetition compared with precompetition. There was less than 2 hours between the precompetition and postcompetition testing to minimise the circadian variations in hormone release. ${ }^{25}$ Larger time slots may not be sensitive enough to detect differences between precompetition an postcompetition measurements. This may explain the contrary findings in a study conducted in a group of male athletes $(n=19)$ providing a saliva sample the morning before and 1 day after ( 24 hours post) an international rugby union match. ${ }^{26}$ In this design, no significant changes in cortisol levels from precompetition to postcompetition were found or reported.

In addition, our third finding showed significantly higher cortisol levels in morning race compared with the levels measured after the afternoon race. This is in agreement with previous research showing that cortisol exhibits a marked circadian rhythm, characterised by a rapid increase in levels on awakening peaking at around $30 \mathrm{~min}$ postawakening and declining thereafter reaching lowest levels in the evening. ${ }^{16} 27-29$

Given the non-significant difference in the lightweight crew between the cortisol concentrations before the finals on Saturday, the early morning prerace sample on Sunday and the samples on Sunday afternoon (in inactivity), we must assume that circadian effects had at best only a mild effect on cortisol concentrations in this small sample.

Consequently, the convincing differences with the racing crew on Sunday afternoon (the heavyweights), already in their prerace cortisol values, can only by explained by either physiological differences between both crews (like potentially the dietary consequences of weight restrictions), or, more likely, mental issues in preparation of the upcoming event.

Finally, comparing the 'normal' cortisol concentrations of the idle lightweight crew on Sunday afternoon with the race values of both crews indicates that at least some proportion of the increase in race-related corticosteroid production is likely to be caused by mental factors. However, the physical demands of a race overshadow this increase impressively: based on our data in a proportion of approximately 1:5.

The present study is to our knowledge the first to assess cortisol concentrations in a highly standardised procedure, both over six consecutive mornings, and previous to, during and immediate after seven rowing races. This was possible as in our study we used multiple saliva samples to assess cortisol concentrations on a relatively high frequency, whereas most previous studies used venous blood samples when measuring cortisol levels in rowers. ${ }^{20-23}$ Salivary cortisol has some considerable advantages compared with blood sampling and therefore, salivary diagnosis is an increasingly important approach in sports medicine ${ }^{29}$ Salivary measurements avoid the stress caused by venipuncture, ${ }^{28} 29$ do not need highly trained assessment professionals, while the validity seems accurate: salivary steroids show equilibrium with blood concentrations and may provide a better measure than serum cortisol of the stress response, as it more accurately measures the amount of unbound cortisol compared with serum measures. ${ }^{29}$

However, some limitations have to be taken into account. Most primarily, despite the sophisticated procedure, the small sample size, including two quite different teams (in terms of physical fitness and mental status), should make us interpret the results with caution. Not least because the interindividual variability in cortisol production is considerable.

Second, comparing cortisol responses directly with other markers of physical strain during the races (eg, ECG derivatives or metabolic indicators) might be relevant for further research. And finally, although the 
trend in early morning cortisol concentrations does suggest that recuperation is well under way 2 days after the competition, given the present data we can of course not be $100 \%$ sure. Future research might want to include more days after competition to ensure a complete overview of the recuperation of the hypothalamic-pituitary-adrenal axis.

Acknowledgements The authors would like to thank the rowers and their coaches for participation in this study. They thank Nelleke van Wouwe and Janine Herweijer for their help in collecting the data, Andrea Jetten for her help in analysing the data, and the Royal Dutch Rowing Association (KNRB) for providing a testing room at the national training centre.

Funding The study described here has been funded using (financial) resources of The Netherlands Organization for Applied Scientific Research (TNO), which were specifically allocated for scientific research.

\section{Competing interests None declared.}

Ethics approval The Institutional Review Board (IRB) of TNO granted ethical approval for the study.

Provenance and peer review Not commissioned; externally peer reviewed.

Open Access This is an Open Access article distributed in accordance with the Creative Commons Attribution Non Commercial (CC BY-NC 4.0) license, which permits others to distribute, remix, adapt, build upon this work noncommercially, and license their derivative works on different terms, provided the original work is properly cited and the use is non-commercial. See: http:// creativecommons.org/licenses/by-nc/4.0/

(C) Article author(s) (or their employer(s) unless otherwise stated in the text of the article) 2017. All rights reserved. No commercial use is permitted unless otherwise expressly granted.

\section{REFERENCES}

1. Rushall BS. A tool for measuring stress tolerance in elite Athletes. $J$ Appl Sport Psychol 1990;2:51-66.

2. Moreira A, Freitas CG, Nakamura FY, et al. Effect of match importance on salivary cortisol and immunoglobulin $A$ responses in elite young volleyball players. J Strength Cond Res 2013;27:202-7.

3. Passelergue $P$, Lac G. Saliva cortisol, testosterone and $T / C$ ratio variations during a wrestling competition and during the postcompetitive recovery period. Int J Sports Med 1999;20:109-13.

4. Banfi G. Hormonal ratio (cortisol: testosterone) as a marker of exhaustion in Athletes. Journal of Sports Medicine and Physical Fitness, 38, 91士 1998;92.

5. Bateup HS, Booth A, Shirtcliff EA, et al. Cortisol, and women's competition. Evol Hum Behav 2002;23:181-92.

6. Filaire E, Sagnol M, Ferrand C, et al. Psychophysiological stress in judo Athletes during competitions. J Sports Med Phys Fitness 2001;41:2632001.

7. Häkkinen K, Pakarinen A, Alén M, et al. Relationships between training volume, physical performance capacity, and serum hormone concentrations during prolonged training in elite weight lifters. Int $J$ Sports Med 1987;8(Suppl 1):S61-5.
8. Häkkinen K, Pakarinen A. Serum hormones in male strength Athletes during intensive short term strength training. Eur J Appl Physiol Occup Physiol 1991;63:194-9.

9. Haneishi K, Fry AC, Moore CA, et al. Cortisol and stress responses during a game and practice in female collegiate soccer players. $J$ Strength Cond Res 2007;21:583-8.

10. Passelergue P, Robert A, Lac G. Salivary cortisol and testosterone variations during an official and a simulated weight-lifting competition. Int J Sports Med 1995;16:298-303.

11. Viru A. Plasma hormones and physical exercise. Int J Sports Med 1992;13:201-9.

12. Viru A, Karelson K, Smirnova T. Stability and variability in hormona responses to prolonged exercise. Int J Sports Med 1992;13:230-5.

13. Buchanan TW, al'Absi M, Lovallo WR. Cortisol fluctuates with increases and decreases in negative affect. Psychoneuroendocrinology 1999;24:227-41.

14. Erickson K, Drevets W, Schulkin J. Glucocorticoid regulation of diverse cognitive functions in normal and pathological emotional states. Neurosci Biobehav Rev 2003;27:233-46.

15. Filaire E, Ferreira JP, Oliveira M, et al. Diurnal patterns of salivary alpha-amylase and cortisol secretion in female adolescent tennis players after 16 weeks of training. Psychoneuroendocrinology 2013;38:1122-32.

16. Wüst $\mathrm{S}$, Wolf $\mathrm{J}$, Hellhammer $\mathrm{DH}$, et al. The cortisol awakening response - normal values and confounds. Noise Health 2000;2:79-88.

17. Elias M. Serum cortisol, testosterone, and testosterone-binding globulin responses to competitive fighting in human males. Aggress Behav 1981;7:215-24.

18. Booth A, Shelley G, Mazur A, et al. Testosterone, and winning and losing in human competition. Horm Behav 1989;23:556-71.

19. Salvador A, Suay F, González-Bono E, et al. Anticipatory cortisol, testosterone and psychological responses to judo competition in young men. Psychoneuroendocrinology 2003;28:364-75.

20. Jürimäe $J$, Jürimäe $T$, Purge $P$. Plasma testosterone and cortisol responses to prolonged sculling in male competitive rowers. $J$ Sports Sci 2001;19:893-8.

21. Jürimäe J, Mäestu J, Purge $P$, et al. Relations among heavy training stress, mood state, and performance for male junior rowers. Percept Mot Skills 2002;95:520-6.

22. Kokalas N, Tsalis G, Tsigilis N, et al. Hormonal responses to three training protocols in rowing. Eur J Appl Physiol 2004;92:128-32.

23. Pearson R, Ungpakorn G, Harrison GA. Catecholamine and cortisol levels in Oxford college rowers. Br J Sports Med 1995;29:174-7.

24. Bille K, Figueiras D, Schamasch $P$, et al. Sudden cardiac death in Athletes: the Lausanne Recommendations. Eur J Cardiovasc Prev Rehabil 2006;13:859-75.

25. Choi JC, Min S, Kim YK, et al. Changes in pain perception and hormones pre- and post-kumdo competition. Horm Behav 2013;64:618-23.

26. Crewther BT, Al-Dujaili E, Smail NF, et al. Monitoring salivary testosterone and cortisol concentrations across an international sports competition: data comparison using two enzyme immunoassays and two sample preparations. Clin Biochem 2013;46:354-8.

27. Hucklebridge $F$, Hussain $T$, Evans $P$, et al. The diurnal patterns of the adrenal steroids cortisol and dehydroepiandrosterone (DHEA) in relation to awakening. Psychoneuroendocrinology 2005;30:51-7.

28. Gura T. Just spit it out. Nat Med 2008;14:706-9.

29. Vining RF, McGinley RA, Maksvytis JJ, et al. Salivary cortisol: a better measure of adrenal cortical function than serum cortisol. Ann Clin Biochem 1983;20 (Pt 6):329-35. 